\title{
Immunolocalization of Aquaporin Water Channels in the Domestic Cat Male Genital Tract
}

\author{
S Arrighi and M Aralla* \\ Laboratory of Anatomy, Department of Health, Animal Science and Food Safety, Università degli Studi di Milano, Milano, Italy
}

\begin{abstract}
Contents
Four different aquaporins (AQP1, 2, 5 and 9), integral membrane water channels that facilitate rapid passive movement of water, were immuno-localized in the excurrent ducts collected from sexually mature cats during orchiectomy. Aquaporins 1, 2 and 9, were immuno-localized at distinct levels, whereas AQP5 was undetectable all along the feline genital tract. No immunoreactivity was present at the level of the rete testis with any of the antibodies tested. In the efferent ducts, AQP1-immunoreactivity was strongly evidenced at the apical surface of the non-ciliated cells, and AQP9-immunoreactivity was shown at the periphery of both ciliated and nonciliated cells. Aquaporins 2 was absent in the caput epididymidis, either in the efferent ducts or in the epididymal duct. Otherwise, AQP2 was increasingly localized at the adluminal surface of principal cells from the corpus to the cauda epididymidis and more weakly in the vas deferens epithelium. The supranuclear zone of the epididymal principal cells was AQP9-immunoreactive throughout the duct, with the exclusion of the vacuolated sub-region of the caput and with higher reaction intensity in the cauda region. AQP1 was present in the blood vessels all along the genital tract. AQP1 was expressed also in the smooth muscle layer of the vas deferens. The tested AQP molecules showed a different expression pattern in comparison with laboratory mammals, primates and the dog, unique other carnivore species studied to date. The present information is possibly useful in regard to the regional morphology of the feline epididymis and correlated functions, which are still a matter of debate.
\end{abstract}

\section{Introduction}

The intratesticular excurrent ducts, consisting of the mediastinic lacunae called rete testis, communicate with the extratesticular rete by a short tunical portion traversing the albuginea. Here, transition through the urogenital junction leads to the efferent ducts (Wrobel and Gurtler 2004), a highly absorptive portion. Thereafter, the very elongated epididymal duct follows, whose proximo-distal histo-physiologic variations have been a matter of discussion for a long time in the different animal species. At its termination, the conduit becomes the straight and muscular vas deferens, which leaves the scrotum as a component of the spermatic cord. Far from being unvarying conduits, the different portions of the excurrent ducts are environments with highly specialized regional differentiation at the basis of modulated secretory, absorptive and contractile functions contributing the sperm maturation taking place in the lumen. This process may require different microenvironments in relation to specific needs and implies region-specific gene expression (Belleannée et al. 2012). Actually, even if the general organization of the epithelium lining of mammalian epididymis is almost the same, differences and peculiarities are frequent, mirroring species-specific functional modulations.

Significant water movements take place throughout the duct. In the efferent ducts, more than $80 \%$ of the testicular fluid is reabsorbed (Clulow et al. 1994) and the epithelial absorptive activity continues along the epididymis, turning out in a progressive increase of sperm concentration. Secretory processes occur as well throughout the epididymis, giving rise to perhaps the most complex fluid found in the lumen of any organ involved in secretory functions, resulting from the continuous changes in composition as well as the presence of constituents in unusually high concentrations, or those not present in other body fluids (Cornwall 2009). Recent literature gives considerable importance to the presence of proteins of the aquaporin family at different levels all along the male genital tract, in rodents (Pastor-Soler et al. 2001, 2002, 2010; Badran and Hermo 2002; Hermo et al. 2004, 2008; Oliveira et al. 2005; Da Silva et al. 2006a,b; Picciarelli-Lima et al. 2006; Arrighi et al. 2010a; Hermo and Smith 2011), primates (Fisher et al. 1998) and, sporadically, carnivores (Domeniconi et al. 2007, 2008; Arrighi et al. 2010b). Aquaporins (AQPs) are a class of small, hydrophobic, integral membrane proteins that facilitate rapid passive movement of water (Agre et al. 2002). Aquaporins are commonly composed of four identical subunit proteins in mammals, with each monomer acting as a channel through which water may pass (Gonen and Walz 2006). Water molecules are tied oneby-one and then released permitting the crossing through the porus in the opposite senses, driven by an osmotic gradient through the membrane. Thus, an important characteristic of AQPs is bi-directionality. Extremely diffused in nature, in plants, bacteria or several members of the animal kingdom, 13 AQPs have been identified in mammals, all of them highly permeable to water. Aquaporins 3, 7, 9 and 10 are also permeable to glycerol and some small solute and are known as aquaglyceroporins. Water handling by AQPs in female and male genital systems is crucial for reproduction, and their manifold functions were recently reviewed (Huang et al. 2006; Zhang et al. 2012).

Many questions are still open about male excurrent ducts, among them: the interactions between lumen wall and lumen content are species-specific as are, of course, the gametes and their maturation process taking place in the epididymal microenvironment; proximo-distal modulations of excurrent duct morpho-physiology are strictly species-related, as well; the link between morphology and function is an always present subject of 
discussion (Cornwall 2009; Belleannée et al. 2012; Dacheux et al. 2012). In the light of these considerations, the purpose of the present work was to add information to the study of the whole male genital tract - from the rete testis to the vas deferens - in the feline species, investigating the possible fluid exchanges taking place at epithelial level by means of the immunohistochemical localization of three different aquaporins (AQP1, 2 and 5) and one aquaglyceroporin (AQP9), whose presence has been extensively studied in the epididymal epithelium of the species studied up to date (Fisher et al. 1998; Pastor-Soler et al. 2001, 2002, 2010; Badran and Hermo 2002; Oliveira et al. 2005; Da Silva et al. 2006a,b; Picciarelli-Lima et al. 2006; Domeniconi et al. 2007, 2008; Hermo et al. 2008). Although the water-transporting mechanism through membraneanchored proteins is an evolutionary conserved process, the expression patterns of several AQPs have been shown to display inter-species variations. Thus, it is challenging extrapolating data obtained from one species to another. Moreover, the likely differential expression might also reflect diversified microenvironments according to species.

The results will be put in relation and discussed in the light of the specific regions described in the domestic cat epididymis (Axnér et al. 1999) and the cell types that constitute the epithelium, whose ultrastructural features strongly indicate the involvement in resorption as well as secretory functions (Arrighi et al. 1986).

\section{Materials and Methods}

Epididymides were obtained from five privately owned healthy cats during castration surgery realized in the Reproduction Unit of the faculty of Veterinary Medicine of the University of Milan, Italy. Cats were aged 8 months, 11 months and 1,2 and 3 years. As it is known that moderate seasonality exists in the testis function of the domestic cat (Axnér and Linde Forsberg 2007; Blottner and Jewgenow 2007), collection of samples was homogeneously performed in FebruaryMarch. Fragments of testis, epididymis and scrotal vas deferens were collected and immediately immersed in fixative. Histological examination of testicular tissues was aimed at verifying the sexual maturity of all the cats, especially the younger ones. Thanks to the small dimensions, one of the two gonads of each cat was fixed in toto with the associated epididymis. The contralateral epididymis was gently dissected free from the testis, fixed and processed in toto and longitudinally embedded. The two different modes of sampling were aimed to obtain information as complete as possible about the connections of the different parts of the intrascrotal genital tract - from the rete testis to the vas deferens - to each other and to pick out possible regional modulations apart from the classic subdivision in caput, corpus and cauda. Pieces were fixed in formalin $10 \%$ for $24-48 \mathrm{~h}$ at $4^{\circ} \mathrm{C}$. After fixation, fragments were dehydrated in a graded series of ethanol, clarified in xylene and embedded in paraffin. Serial sections were cut at $4 \mu \mathrm{m}$ thickness, de-waxed and stained with routinary haematoxylin and eosin (HE) for general morphological purposes. Other sections were mounted onto poly-L-lysine-coated slides, de-waxed and used for the immunohistochemical procedures according to previously described methods (Aralla et al. 2009, 2012; Arrighi et al. 2010a,b). Sections of all specimens and controls included in the study were simultaneously processed in the same session of immunohistochemistry. Antibodies, buffer and revelation solutions were made fresh for each run. Trisbuffered saline (TBS: $0.05 \mathrm{~m}$ Tris $/ \mathrm{HCl}, 0.15 \mathrm{M} \mathrm{NaCl}$ ) buffer was used for rinses throughout the whole procedure.

Briefly, sections were washed and immersed in a freshly prepared $3 \% \mathrm{H}_{2} \mathrm{O}_{2}$ solution for 15 min to block the endogenous peroxidase activity, followed by incubation in 1:20 normal goat serum (DakoCytomation, Glostrup, Denmark) in TBS for $30 \mathrm{~min}$ to prevent background prior to incubation with primary antiserum. Sections were then incubated overnight in a humidity chamber at room temperature using rabbit polyclonal antibody against rat Aquaporin 1, Aquaporin 2, Aquaporin 5 and Aquaporin 9 (Alpha Diagnostic International, San Antonio, TX,USA; Cat \#, respectively, AQP11-A, AQP21-A, AQP51-A and AQP91-A). AQP1- and AQP2-antisera were diluted 1: 100 (final concentration $10 \mu \mathrm{g} / \mathrm{ml}$ ), AQP5-antiserum 1 : 30 (final concentration $33 \mu \mathrm{g} / \mathrm{ml}$ ) and AQP9-antiserum $1: 300$ (final concentration $3.3 \mu \mathrm{g} / \mathrm{ml}$ ) in specific antibody diluent (DakoCytomation, code S302283).

The sections designed to be incubated with AQP5 antibody were pre-treated with two microwave cycles $(2 \times 5 \mathrm{~min}$ at $450 \mathrm{~W}$ in $0.01 \mathrm{M}$ citrate buffer, $\mathrm{pH}$ 6.0) to unmask the antigen. The sections were then washed and incubated for $30 \mathrm{~min}$ with goat anti-rabbit immunoglobulins (DakoCytomation) diluted 1:200. Either streptavidin-biotin complex (StrepABComplex/HRP, DakoCytomation) or Envision System Labelled Polymer-HRP (DakoCytomation) were employed as revelation system. Immunoreactive sites were visualized using a freshly prepared solution of $4 \mathrm{mg} \mathrm{3.3'-diaminobenzi-}$ dine tetrahydrochloride (DAB; Sigma-Aldrich, St. Louis, MO, USA) in $10 \mathrm{ml}$ of a $0.5 \mathrm{~m}$ Tris buffer at $\mathrm{pH} 7.6$ containing $0.1 \mathrm{ml}$ of $3 \% \mathrm{H} 2 \mathrm{O} 2$ for $13-20 \mathrm{~min}$. Sections were counterstained with Mayers' haematoxylin, dehydrated and mounted using Eukitt ${ }^{\circledR}$ (Bio-Optica, Milan, Italy).

Although the antibodies employed in this study are directed towards peptides from rat AQPs, a high percentage of homology exists between rat and cat AQPs. The peptide for the AQP1 antibody directed towards peptides from rat is $90 \%$ homologous for cat, the one for AQP2 is $82 \%$ homologous, the one for AQP5 is $64 \%$ homologous, and the one for AQP9 is $80 \%$ homologous (data from http://www.uniprot.org). Moreover, the effectiveness of all the antibodies has been previously demonstrated on canine and feline tissues (Aralla et al. 2009, 2012; Arrighi et al. 2010b), in the rat epididymis (Arrighi et al. 2010a) and ovine salivary gland (Scocco et al. 2011). Sections of rat organs similarly processed as above served as positive controls for AQP1 (kidney, Fig. 2g inset), AQP2 (kidney, Fig. 3b inset), AQP5 and AQP9 (liver, Fig. $4 \mathrm{~h}$ inset) antisera. The specificity of the immunostaining was tested by including negative controls, performed by (i) use of non-immune rabbit serum (DakoCytomation; 
code \# X0903) in place of specific antisera and (ii) omission of the primary antibody. No immunoreactivity was seen in the control preparations (insets in Figs $2 \mathrm{f}$ and $4 \mathrm{~g})$.

Assessment of the immunostainings was based on subjective estimates of both authors. Double-blind evaluation of the sections included staining intensity, congruity in staining between duplicate or triplicate samples obtained from the different animals, validation of immunohistochemical staining in comparison with positive and negative controls, and localization of the immunoreactivities (cytoplasmic or in the cell membranes). Slides were observed and photographed under an Olympus BX51 photomicroscope using UPlan Apo $4 \times / 0.16$ NA, UPlan Apo $10 \times / 0.40$ NA, UPlan Apo $20 \times /$ 0.70NA, UPlan Fi $40 \times / 0.70 \mathrm{NA}$ and UPlan Fi $100 \times$ $1.30 \mathrm{NA}$ oil lenses. Photomicroscope was equipped with a digital camera Olympus C-5060 Wide Zoom and DP software (Olympus, Italy) for computer-assisted image acquirement and managing.

\section{Results}

Histological evaluation of the testis morphology showed active spermatogenesis in all the specimens, demonstrating that the cats employed in this study were all sexually mature. Also, histological and immunohistochemical results were basically the same in cats of different ages.

In suitable HE-stained sections, it was possible to see the different components of the rete testis: the anastomosed intratesticular rete and, continuous with it and juxtaposing to the extremitas capitata of the testis, the extratesticular part (Fig. 1a,b). In continuity with this, the efferent ducts abruptly started, with a change in the epithelial cover from low cuboidal in the rete to simple columnar epithelium in the efferent ducts (Fig. 1b,c). From that deep inner point of the efferent ducts (proximal portion) to the part more distant from the testis (distal portion), the wide lumen of the ductuli became narrower, while the epithelium became taller (Fig. 1b). Along with this passage, the epithelium did not change its characteristics, that is, the presence of two cell types, ciliated and non-ciliated cells, which apparently remained unvaried (Fig. 1d). Efferent ducts occupied a part of the caput epididymidis. The remaining part was occupied by the epididymal duct, which showed sub-regions with different morphology (Fig. 1e). In the initial segment (is in Fig. 1e), the tubule had a large diameter and the lumen was wide and generally empty. In the subsequent region, the epithelial cells had a conspicuous cytoplasmic vacuolization ( $v r$ in Fig. 1e),

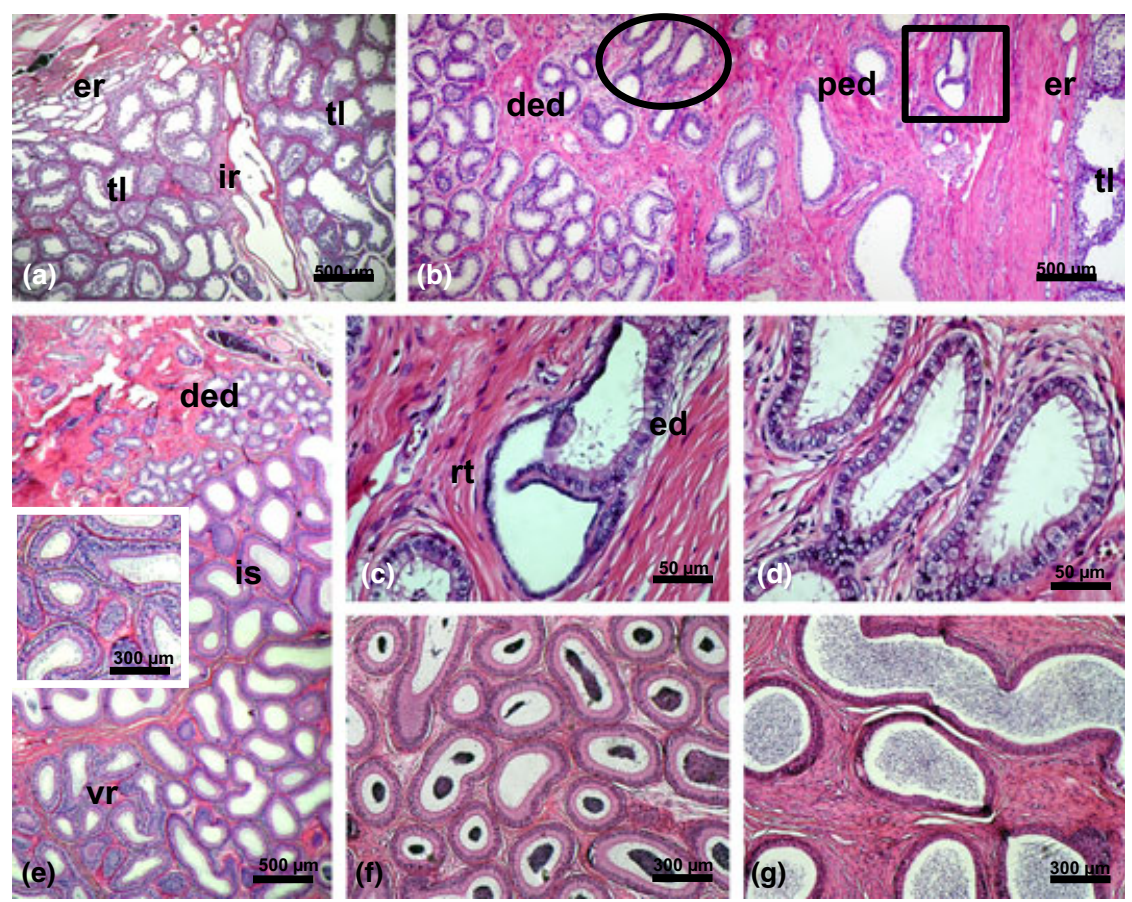

Fig. 1. Genital tract morphology in the adult cat. Haematoxylin and eosin stain. (a) The intratesticular (ir) and the extratesticular rete testis (er) can be seen. The intratesticular anastomosed network of canals of the rete is located in the mediastinum testis, surrounded by testicular lobules (tl) on both sides. (b) At the extremitas capitata of the testis, it is possible to see the large lacunae of the extratesticular rete testis (er), which extend at the periphery of testicular lobules (tl) immersed into an abundant stroma. The proximal (ped) and distal (ded) components of the efferent ducts can also be seen. A tubular section showing the continuity between the rete and an efferent duct is framed into a square and is shown at higher magnification in (c). Area framed into an oval is shown at higher magnification in (d). (c) Detail of abrupt passage from the low cuboidal epithelium lining the rete testis (er) to the simple columnar epithelium lining the efferent ducts (ed). (d) The columnar epithelium lining the efferent ducts is made up by ciliated and non-ciliated cells. (e) The cat caput epididymidis is occupied in part by the distal efferent ducts (ded). The remaining part is occupied by the epididymal duct, showing two sub-regions with different morphology (is: initial segment; vr: vacuolated region). The epididymal duct shows a wide lumen lined by columnar epithelium in both sub-regions, but in the vacuolated region (vr), epithelial cells have a foamy cytoplasm, as shown in the inset at higher magnification. (f) In the corpus epididymidis, plenty of spermatozoa can be seen in the lumen, which is covered by pseudostratified epithelium. (g) In the cauda epididymidis, sperm are more concentrated into the lumen and the duct epithelium is lower 


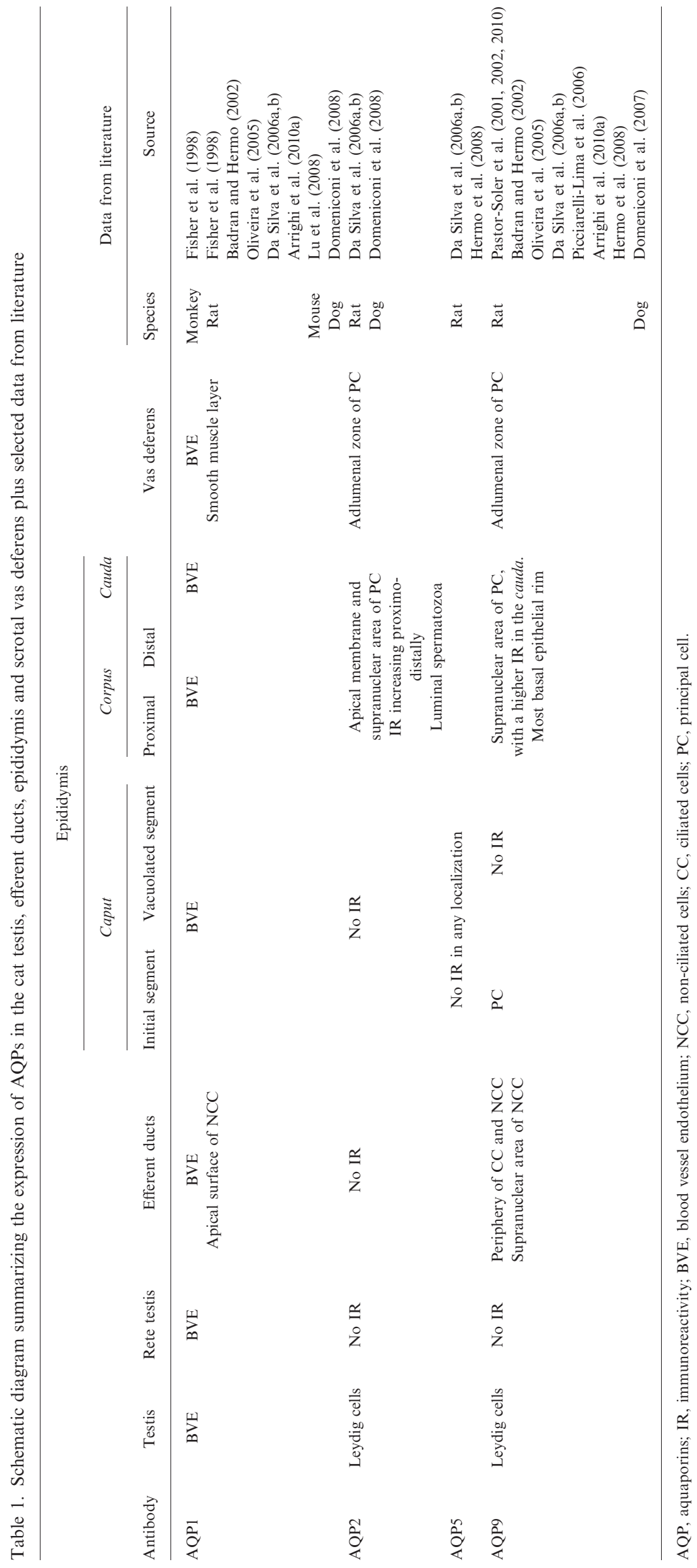




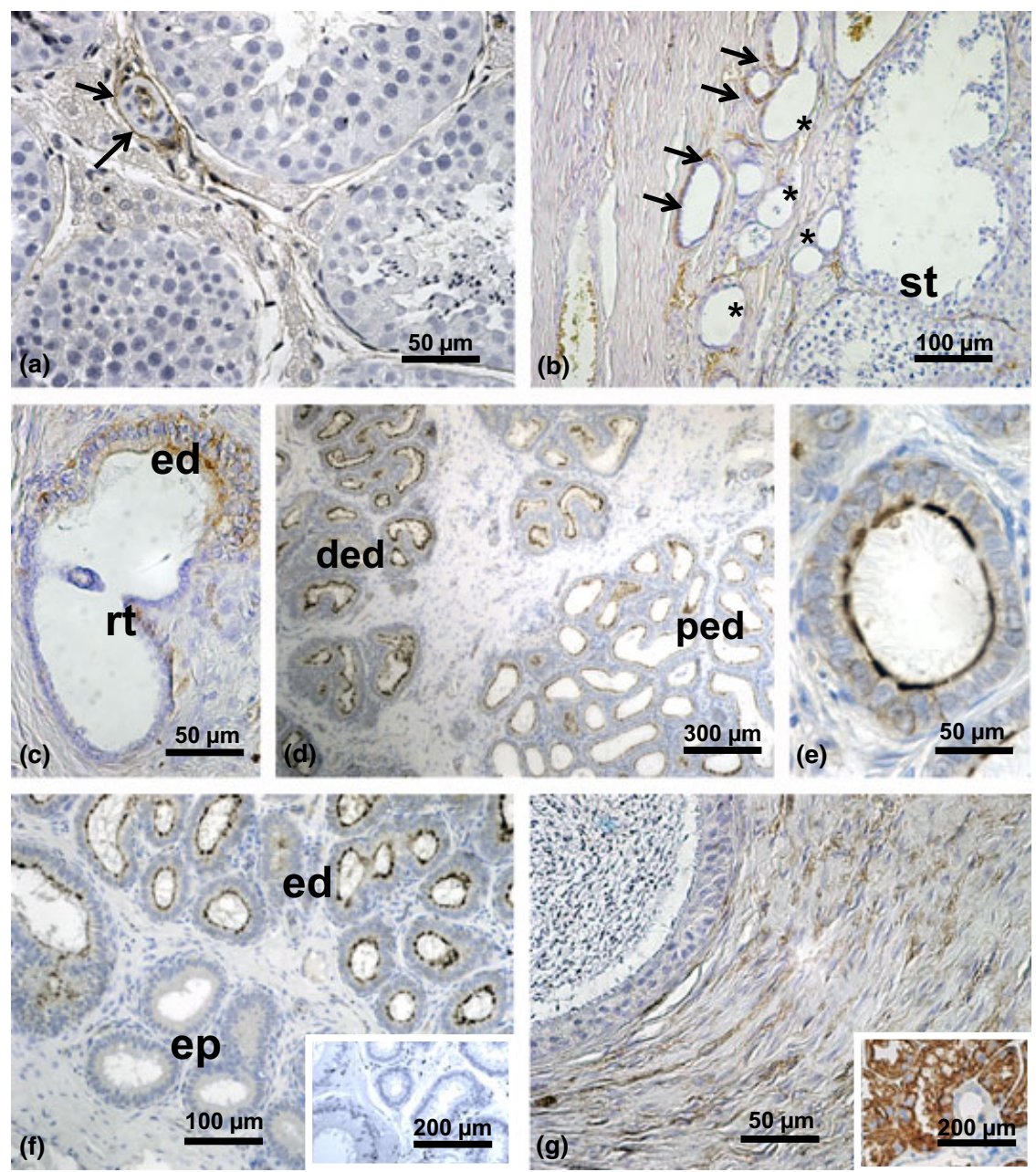

Fig. 2. Aquaporin-1 immunohistochemistry. (a) AQP1-immunoreactivity is evident in the endothelium of the blood vessels in the testicular interstitium. At the level of the arteries (arrows), AQP1-immunoreactivity is present also in the adventitia. (b) At the extremitas capitata of the testis, it is possible to notice the absence of AQP1-immunoreactivity at the level of the seminiferous tubules (st) and rete testis (asterisks). Arrows indicate immunoreactive epithelial cells lining the proximal part of the efferent ducts. Red blood cells are positive, too. (c) At the limit between the rete testis and the efferent duct, the epithelium lining the efferent duct (ed) shows AQP1-immunoreactivity, whereas the epithelium lining the rete testis (rt) is unstained. (d) AQP1-immunoreactivity is stronger in the epithelium lining the distal components of the efferent ducts (ded) than in the proximal ducts (ped). (e) In the efferent ducts, strong reaction is present at the apical surface of the non-ciliated cells corresponding to the microvilli, whereas the ciliated cells remain unstained. A diffuse reaction can be seen in the lateral plasma membranes of adjacent epithelial cells. (f) In the caput region, evident AQP1-immunopositivity can be seen at the apical surface of the epithelium lining the efferent ducts (ed). In contrast, the epididymal epithelium (ep) is unreactive. Inset: Negative control obtained by use of non-immune rabbit serum in place of the primary antibody. (g) In a section of scrotal vas deferens, AQP1-immunoreactivity is detectable at the level of the smooth muscle layer. Inset: Adult rat kidney utilized as positive control for AQP2-immunoreaction. Strong membrane and cytoplasmic immunostaining can be seen on cells lining the proximal convoluted tubules

and duct diameter was smaller and the lumen narrower (see also inset in Fig. 1e). At the corpus level, spermatozoa were present in a large number into the lumen (Fig. 1f). The duct had the widest diameter at the cauda level, where a huge number of spermatozoa were present in the lumen (Fig. 1g). The epithelium was consistently lower in more distal regions, where the smooth muscle layer surrounding the duct started to thicken.

The scrotal vas deferens had a narrow lumen lined by pseudostratified columnar epithelium with short stereocilia. A very thick muscular sheath was made up by three concentric layers of smooth muscle.

\section{AQP-immunoreactivity}

A different immunoreactivity was noticed with the four antisera tested, with AQP-specificity varying in the diverse regions of the male excurrent duct and differently localized in the cell structures. Table 1 summarizes the expression of AQPs in the cat testis, efferent ducts, epididymis and scrotal vas deferens.

Aquaporins 1-immunoreactivity decorated the blood vessel endothelium at testicular level (Fig. 2a) and all along the genital tract. Aquaporins 1-immunoreactivity, strongly evidenced at the apical surface of the efferent ducts non-ciliated cells (Fig. 2c-f), was absent from the epithelium of the rete testis (Fig. 2b,c), epididymis (Fig. 2f) and vas deferens (Fig. 2g). Aquaporins 1 was expressed in the smooth muscle layer of the vas deferens (Fig. 2g).

Aquaporins 2-immunoreactivity was detected at testicular level, localized in the interstitial Leydig cells (Fig. 3a). Reactivity was absent in the caput epididymidis, either in the efferent ducts (Fig. 3b) or in different 

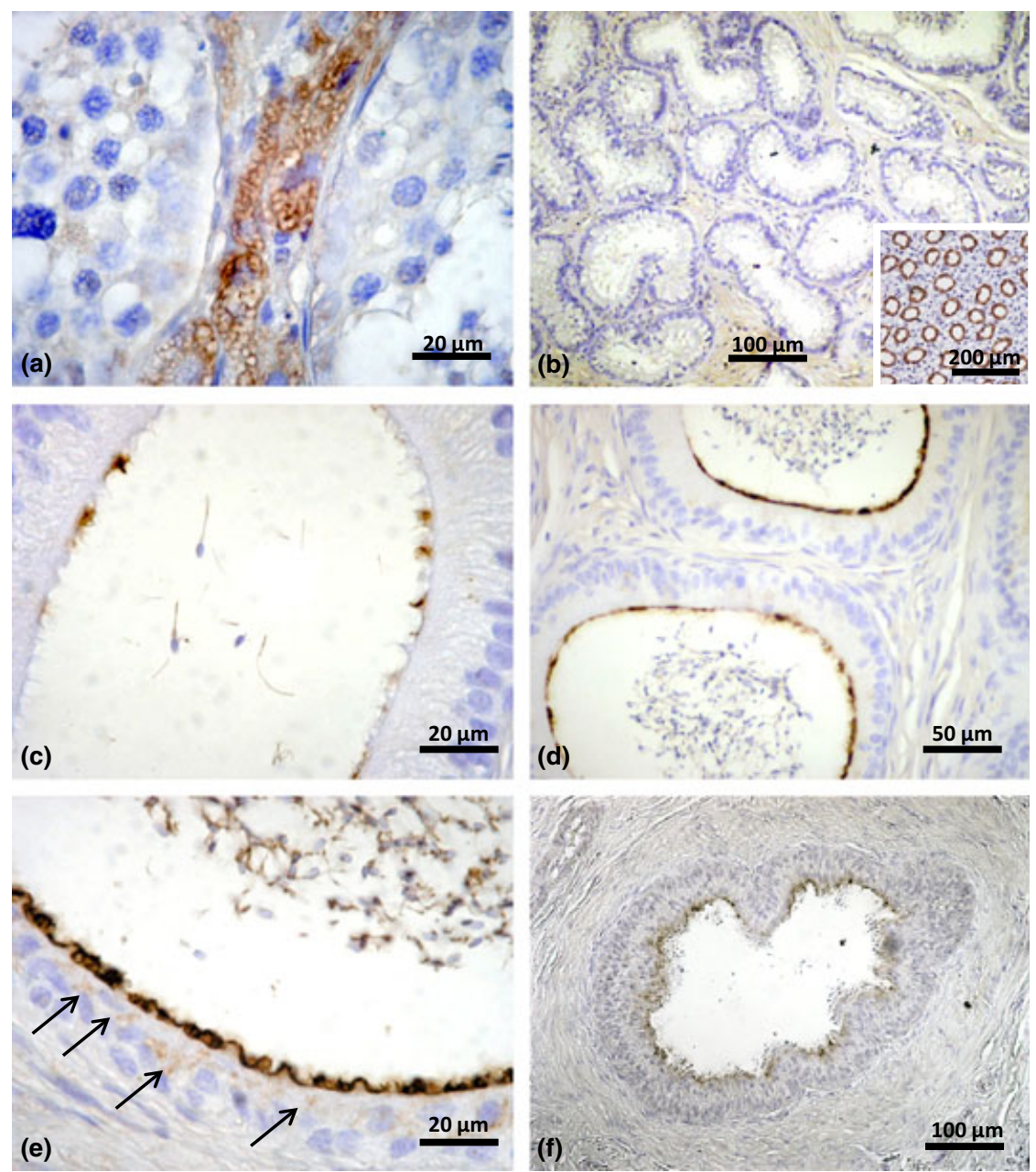

Fig. 3. Aquaporin-2 immunohistochemistry. (a) AQP2-immunoreactivity is strongly expressed in the interstitial Leydig cells of the testis. (b) No reactivity is detectable in the efferent ducts. Inset: Adult rat kidney utilized as positive control for AQP2-immunoreaction. Strong immunostaining can be seen on the plasma membranes of the epithelial cells lining the collecting tubules. (c) In the more proximal corpus epididymidis, AQP2-immunoreactivity can be seen at the apical cell membrane and microvilli of rare columnar cells. (d) More numerous AQP2immunoreactive cells were noticed in the distal corpus. (e) In the cauda epididymis, the totality of the principal cells shows intense positivity at the most apical surface. Weak immunoreactivity can be noticed also at the level of the supranuclear area of the cells (arrows), and in the luminal spermatozoa, at the level of the tail membrane. (f) In the scrotal vas deferens, AQP2-immunoreactivity can be seen in the adlumenal zone of the epithelial cells

regions of the epididymal duct. Aquaporins 2-immunoreactivity was evident at the apical cell membrane and microvilli of sporadic columnar cells of the more proximal corpus (Fig. 3c) and increased from the distal corpus (Fig. 3d) up to the cauda epididymis, where the totality of the principal cells showed intense positivity at the most apical surface (Fig. 3e). A weaker immunoreactivity was also noticeable at the level of the supranuclear area of the cells (Fig. 3e). Luminal spermatozoa were AQP2-immunoreactive, too, at the level of the tail membrane (Fig. 3c-e). The adlumenal zone of the epithelium was also immunoreactive in the scrotal vas deferens (Fig. 3f).

Aquaporins 9 aquaglyceroporin was detected at testicular level, localized in the interstitial Leydig cells (Fig. 4a). No immunoreactivity was present at the level of the rete testis (Fig. 4a,b), but it suddenly started with the beginning of the efferent ducts (Fig. 4b). Aquaporins 9-immunoreactivity was shown at the periphery of both ciliated and non-ciliated epithelial cells and at the supranuclear area of the non-ciliated cells, principally
(Fig. 4b,c). At epididymal level, the supranuclear zone of the principal cells was immunoreactive throughout the duct, with a higher intensity of reaction in the cauda region (Fig. 4d,f,g). Aquaporins 9-immunoreactivity was present in the supranuclear and apical cytoplasm and in the most basal epithelial rim (Fig. 4h). However, there was a region of the epididymis where no reactivity could be detected, that is, the vacuolated sub-region of the caput (Fig. 4e).

Aquaporins 5-immunoreactivity was undetectable (even after antigen retrieval pre-treatment), despite the reactivity shown in salivary gland sections utilized as positive control.

\section{Discussion}

This study pointed out some aspects of the morphology of the intratesticular genital tract and investigated by immunohistochemistry the expression of four aquaporin proteins in the excurrent ducts of the domestic tom cat. 


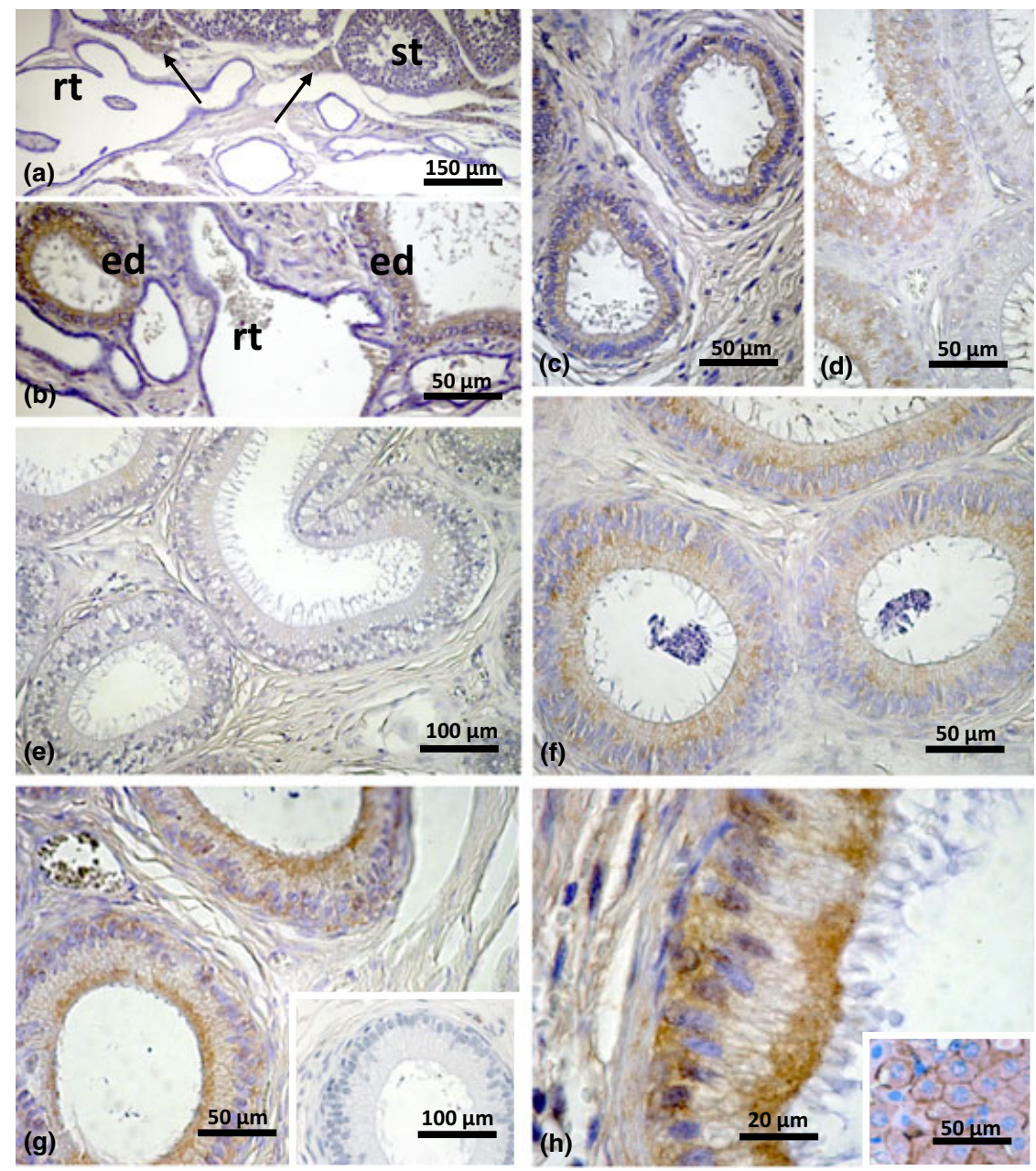

Fig. 4. Aquaporin-9 immunohistochemistry. (a) Testicular Leydig cells show a moderate AQP9-immunoreactivity (arrows). No reactivity is present in the epithelium lining the rete testis (rt). (b) Moderate (on the right)-to-strong (on the left) AQP9-immunoreactivity is present in the epithelium lining the more proximal part of the efferent ducts (ed), next to the rete testis (rt), which is unreactive. (c) AQP9-immunolocalization is evident at the apical and lateral cell membrane of epithelial cells lining cat efferent ducts, as well as in the apical cytoplasm and sporadically in the more basal cellular rim towards the basal lamina. (d) In sub-region I of the caput epididymidis, weak AQP9-immunoreactivity can be seen in the cytoplasm of principal cells. (e) No reactivity can be detected in sub-region II of the caput. (f) In the corpus epididymidis, the supranuclear area of the principal cells shows AQP9-immunoreactivity. (g) In the cauda epididymidis, stronger AQP9-immunoreactivity is present in the apical cytoplasm of principal cells. AQP9-immunoreactivity is also present in the basal epithelial edge. Inset: Negative control obtained by omission of the primary antibody. (h) AQP9-immunoreactivity is localized in the supranuclear and apical cytoplasm of principal cells and in the most basal epithelial rim. Inset: Adult rat liver utilized as positive control for AQP9-immunoreaction. Strong membrane immunostaining can be seen on cells lining the hepatocytes

The histologic survey confirmed in part the data collected by Wrobel and Gurtler (2004) about the feline urogenital junction. Different components of the rete testis were described (intratesticular and extratesticular rete) and, in continuity with it, the efferent ducts started abruptly, with a change in the epithelial cover from low cuboidal in the rete to simple columnar epithelium in the efferent ducts. A slight change was also observed from the proximal to the distal portion of the ductuli efferentes, evidenced by narrowing of the lumen lined by a higher epithelium. Along with this passage, the epithelium did not change its characteristics, that is, the presence of two cell types: ciliated and non-ciliated cells, which apparently remained unvaried.

As concerns the epididymal duct, the histologic results confirmed the regionalization furnished by Axnér et al. (1999). Moreover, peculiarities were found related to the vacuolated region of the caput, linked to AQP expression.

Although much studied in laboratory mammals and sporadically in primates (see literature in Table 1), aquaporin expression is an almost neglected item in the male excurrent duct of domestic animal species, studied before only in the dog (Domeniconi et al. 2007, 2008) and cat (Arrighi et al. 2010b).

Aquaporins to be tested were chosen among the most represented in epididymal epithelium of the species studied up to date. Three of those, namely AQP1, 2 and 9, were differently immuno-localized at distinct levels, whereas AQP5 was immunohistochemically undetectable all along the feline genital tract (Table 1).

Although an influence of age on semen parameters has been reported (Mota and Ramalho-Santos 2006), no striking difference was noticed as concerns the results obtained in mature vs pubertal cats. 
None of the AQPs molecules tested immuno-localized in the cat rete testis. Absence of AQP1 and AQP9 in the cat rete testis confirms previous results obtained in the rat (Fisher et al. 1998; Oliveira et al. 2005). Aquaporins 1 was otherwise localized on the epithelium lining the rete testis in the mouse ( $\mathrm{Lu}$ et al. 2008), marmoset (Fisher et al. 1998) and adult dogs, together with AQP2 (Domeniconi et al. 2008). Maybe that water trafficking in the cat rete testis might be regulated by AQP molecules other than those tested in this study. There is general agreement in the literature on the presence and function of AQP molecules in the efferent ducts. Aquaporins 1- and AQP9-immunoreactivity occur in all the species studied on the microvilli of non-ciliated cells (Pastor-Soler et al. 2001; Badran and Hermo 2002; Oliveira et al. 2005). In adult dogs, AQP1, 2 and 9 are expressed in efferent ducts (Domeniconi et al. 2007, 2008). Rete testis and efferent ductules are embryologically distant organs (Wrobel and Gurtler 2004), being efferent ducts the only tract of the excurrent ducts embryologically related to renal proximal tubules, which absorb up to $80 \%$ of the glomerular ultrafiltrate and where AQP1 is maximally expressed (Schnermann et al. 1998). In the efferent ducts, this water channel is of greatest importance in the concentration of testicular fluid, which requires rapid reabsorption (Clulow et al. 1998). Overabundance of AQP expression in the efferent duct, that is, localization also of AQP9, could be explained by the slight difference in AQP functions, especially when an aquaglyceroporin is implicated in addition to an aquaporin, stressing the overall importance of AQPs in a given tissue (Hermo et al. 2004). Aquaporins 9 at the level of the efferent ducts might allow the passage of glycerol, which has been proposed to serve as a metabolic substrate for sperm to produce $\mathrm{CO}_{2}$ (Cooper and Brooks 1981; Da Silva et al. 2006a).

At epididymal level, AQP expression was detected in the epithelial lining. Aquaporins 2 was absent in the caput epididymidis but was increasingly localized at the adluminal surface of principal cells from the corpus to the cauda epididymidis and more weakly in the vas deferens epithelium. Increased proximo-distal AQP-2 immunoreaction through the epididymis is in contrast with the hypothesis of a possible implication in fluid absorption from the lumen, as it is well known that this function happens in the more proximal epididymal regions. Thus, different, still unknown, functional roles have to be invoked for this species-specific AQP2immunoreactivity. Aquaporins 2-immunoreactivity in the supranuclear area of the principal cells in the cauda region of the epididymal duct might be consistent with PAS-positive granules, which were described in this area correlated, in turn, to few signs of pinocytosis in this specific zone (region 5) of the epididymis (Axnér et al. 1999).

The supranuclear zone of the principal cells was AQP9-immunoreactive throughout the epididymal duct, with the exclusion of the vacuolated sub-region of the caput and the highest intensity of reaction in the cauda region. These are possibly useful information in regard to the regional morphology of the feline epididymis and correlated functions, much debated in the past and still controversial. Remarkable increase in AQPs expression was noticed in the cauda region in the rat, suggestive of major water movement in this region compared with more proximal ones (Hermo and Smith 2011). Nevertheless, as the regional modulations vary tremendously among species, results from one species cannot be assumed to infer regional functions in another. It should also be noticed that some of the rat epididymal cell in which AQP molecules are differently expressed (Hermo and Smith 2011), namely clear cells and narrow cells, are not present in the cat epididymis, which is composed solely by principal and basal cells plus sporadic apical cells and migrating lymphocytes (Arrighi et al. 1986).

Axnér et al. (1999) reported the presence of six different regions based on morphometric results and consistent with their histological differences. Four of the six regions were identified in the caput. The first of them was identified as the initial segment, previously described by Glover and Nicander (1971). The successive regions $2-4$, whose most conspicuous characteristic is the supranuclear vacuolization, were termed the proximal, intermediate and distal parts of the middle segment. It is clear from our results that the vacuolated segment of the feline caput epididymidis shows a different pattern of AQP expression, mirroring some functional, still unknown, characteristic. Peculiar and modulated processes of synthesis and secretion were attributed to the regions of the cat caput epididymis, with accumulation of the products in the duct lumen where they are important for maturation of the spermatozoa (Axnér et al. 1999).

Aquaporins 9 is recognized as the primary aquaporin in epididymis (Pastor-Soler et al. 2010). The increasing AQP9 expression in the cat epididymis and the absence in the epithelium lining the vacuolated segment are possibly indicative of a function not massively related to water absorption, known to take place in the proximal tracts, but to exchanges of solutes with the lumen, which contains perhaps the most complex fluid found in any organ involved in secretory function. Interestingly, one of the solutes that can permeate through AQP9 is glycerol, a spermatozoa metabolic substrate that accumulates in the lumen of the distal epididymis (PastorSoler et al. 2010). It is noteworthy that in the cat cauda epididymidis, where AQP9 was maximally expressed, immunoreactivity was localized in the supranuclear and apical cytoplasm of principal cells and in the most basal epithelial rim, but not in epididymosomes projecting into the lumen. Actually, these blebs are interpreted as membranous vesicles containing proteins and originating from principal cells by apocrine secretion (Cornwall 2009), a process that evidently does not involve AQP presence. The majority of the exosome-like vesicles originate from the cauda epididymal region (Gatti et al. 2005).

No AQP expression was present in the muscular coat of the epididymal duct, while AQP1 expression was reported in this localization in the rat (Oliveira et al. 2005; Arrighi et al. 2010a). In the cat, AQP1-immunoreactivity was detected in the muscular coat only at the level of the vas deferens where it could account for the likely trophic role necessary to rapid contractile cell activities. Aquaporins 2 expression detected in the epithelium lining the vas deferens was noticed also in 
normal mice and rats (Nelson et al. 1998). In the dog (Domeniconi et al. 2008), a positive AQP2-immunoreaction was found in the apical membrane of the epithelial cells in the corpus and in the cauda epididymidis, similarly to our results in the cat, but not in vas deferens. The function attributed to AQP2 in the epididymis epithelium is still uncertain, whereas the presence of AQP2 in the vas deferens was supposed to reflect a function in modifying the luminal fluid content in a hormone-sensitive manner (Nelson et al. 1998).

The AQP1-immunoreactivity of blood vessel endothelia, described also in the rat (Badran and Hermo 2002) and dog (Domeniconi et al. 2008), has an obvious implication in water transport in the intertubular spaces. In tracts where the epithelium is strongly involved in reabsorption processes, that is, the efferent ducts, blood vessel wall might collaborate in water removal from the interstices.

It must be noticed that AQPs show some different expression pattern in the two carnivore species studied to date, the dog (Domeniconi et al. 2007, 2008) and the cat. On the other hand, morpho-physiology of the genital tract in the two species is not always superimposable. It is known that a differential expression of oestrogen receptors alpha and beta is present in the reproductive tracts of adult male dogs and cats (Nie et al. 2002; Schön et al. 2009). In turn, this might affect the expression of AQP molecules, which is known to be regulated by the estrogens (Fisher et al. 1998; Oliveira et al. 2005; Picciarelli-Lima et al. 2006; Pastor-Soler et al. 2010).

As a concluding remark, it can be confirmed that the organization of the epididymal epithelium shows slight species-specific peculiarities, which may mirror different functional modulations related to the sperm maturation occurring in the lumen, a process that might need a diversified microenvironment according to the species.

Further knowledge on the cat epididymal morphophysiology might lead to implementation of gamete preservation and in vitro fertilization protocols, thus increasing the possibilities of assisted reproductive techniques or the opposite aim of developing immunocontraceptive vaccines for permanent non-surgical sterilization of feral cat populations (Munks 2012). The domestic cat is also the suitable model for the study of basic reproductive mechanisms and spermatogenesis regulation, with important rebounds in the control of reproduction of endangered wild felids (Farstad 2000; Amstislavsky et al. 2012).

\section{Acknowledgements}

The Reproduction Unit of the Faculty of Veterinary Medicine of the University of Milan is greatly acknowledged for providing the specimens after cat castration surgery. Grant sponsor: Università degli Studi di Milano.

\section{Conflict of interest}

None of the authors have any conflict of interest to declare.

\section{Author contributions}

S.A. and M.A conceived and designed the experiment and planned the immunocytochemical study. M.A. collected and processed the feline specimens and carried out the immunocytochemical procedures. Both authors evaluated and photographed the slides. S.A. arranged the figures and wrote the manuscript. Both Authors participated in critical reading and final approval of the manuscript.

\section{References}

Agre P, King LS, Yasui M, Guggino WB, Ottersen OP, Fujiyoshi Y, Engel A, Nielsen S, 2002: Aquaporin water channelfrom atomic structure to clinical medicine. J Physiol 542, 3-16.

Amstislavsky S, Lindeberg $\mathrm{H}$, Luvoni $\mathrm{G}$ 2012: Reproductive technologies relevant to the genome resource bank in Carnivora. Reprod Domest Anim 47, 164-175.

Aralla M, Borromeo V, Groppetti D, Secchi C, Cremonesi F, Arrighi S, 2009: A collaboration of Aquaporins handles water transport in relation to the estrous cycle in the bitch uterus. Theriogenology 72, 310-321.

Aralla M, Mobasheri A, Groppetti D, Cremonesi F, Arrighi S, 2012: Expression of aquaporin water channels in canine fetal adnexa in respect to the regulation of amniotic fluid production and absorption. Placenta 33, 502-510.

Arrighi S, Romanello MG, Domeneghini C, 1986: Ultrastructural study on the epithelium lining ductus epididymis in adult cats (Felis catus). Arch Biol 97, 7-24.

Arrighi S, Aralla M, Genovese P, Picabea N, Bielli A, 2010a: Undernutrition during foetal to prepubertal life affects aquaporin 9 but not aquaporins 1 and 2 expression in the male genital tract of adult rats. Theriogenology 74, 1661-1669.
Arrighi S, Ventriglia G, Aralla M, Zizza S Di Summa A, Desantis S, 2010b: Absorptive activities of the efferent ducts evaluated by the immunolocalization of aquaporin water channels and lectin histochemistry in adult cats. Histol Histopathol 25, 433-444.

Axnér E, Linde Forsberg C, 2007: Sperm morphology in the domestic cat, and its relation with fertility: a retrospective study. Reprod Domest Anim 42, 282-291.

Axnér E, Malmqvist M, Linde-Forsberg C, Rodriguez-Martinez H, 1999: Regional histology of the ductus epididymidis in the domestic cat. J Reprod Dev 45, 151160

Badran HH, Hermo LS, 2002: Expression and regulation of aquaporins 1,8 , and 9 in the testis, efferent ducts, and epididymis of adult rats and during postnatal development. J Androl 23, 358-373.

Belleannée C, Thimon V, Sullivan R, 2012 Region-specific gene expression in the epididymis. Cell Tissue Res 349, 717-731.

Blottner S, Jewgenow K, 2007: Moderate seasonality in testis function of domestic cat. Reprod Dom Anim 42, 536-540.

Clulow J, Jones RC, Hansen LA, 1994: Micropuncture and cannulation studies of fluid composition and transport in the ductuli efferentes testis of the rat: comparisons with the homologous metaneph- ric proximal tubule. Exp Physiol 79, 915-928.

Clulow J, Jones RC, Hansen LA, Man SY, 1998: Fluid and electrolyte reabsorption in the ductuli efferentes testis. J Reprod Fertil Suppl 53, 1-14.

Cooper TG, Brooks DE, 1981: Entry of glycerol into the rat epididymis and its utilization by epididymal spermatozoa. J Reprod Fertil 61, 163-169.

Cornwall GA, 2009: New insights into epididymal biology and function. Hum Reprod Update 15/2, 213-227.

Da Silva N, Piétrement C, Brown D, Breton S, 2006a: Segmental and cellular expression of aquaporins in the male excurrent duct. Biochim Biophys Acta 1758, 1025 1033

Da Silva N, Silberstein C, Beaulieu V, Piétrement C, Van Hoek AN, Brown D, Breton S, 2006b: Postnatal expression of aquaporins in epithelial cells of the rat epididymis. Biol Reprod 74, 427-438.

Dacheux JL, Belleannée C, Guyonnet B, Labas V, Teixeira-Gomes AP, Ecroyd H, Druart X, Gatti JL, Dacheux F, 2012: The contribution of proteomics to understanding epididymal maturation of mammalian spermatozoa. Syst Biol Reprod Med 58, 197-210.

Domeniconi RF, Orsi AM, Justulin LA Jr, Leme Beu CC, Felisbino SL, 2007: Aquaporin 9 (AQP9) localization in the adult 
dog testis excurrent ducts by immunohistochemistry. Anat Rec 290, 1519-1525.

Domeniconi RF, Orsi AM, Justulin LA Jr, Leme Beu CC, Felisbino SL, 2008: Immunolocalization of aquaporins 1,2 and 7 in rete testis, efferent ducts, epididymis and vas deferens of adult dog. Cell Tissue Res 332, 329-335.

Farstad W, 2000: Current state in biotechnology in canine and feline reproduction. Anim Reprod Sci 60-61, 375-387.

Fisher JS, Turner KJ, Fraser HM, Saunders PT, Brown D, Sharpe RM, 1998: Immunoexpression of aquaporin-1 in the efferent ducts of the rat and marmoset monkey during development, its modulation by estrogens, and its possible role in fluid resorption. Endocrinology 139, 39353945.

Gatti JL, Métayer S, Belghazi M, Dacheux F, Dacheux JL, 2005: Identification, proteomic profiling, and origin of ram epididymal fluid exosome-like vesicles. Biol Reprod 72, 1452-1465.

Glover TD, Nicander L, 1971: Some aspects of structure and function in the mammalian epididymis. J Reprod Fertil Suppl 13, $39-50$.

Gonen T, Walz T, 2006: The structure of aquaporins. Q Rev Biophys 39, 361-396.

Hermo L, Smith CE, 2011: Thirsty business: cell, region, and membrane specificity of aquaporins in the testis, efferent ducts, and epididymis and factors regulating their expression. J Androl 32, 565-575.

Hermo L, Krzeczunowicz D, Ruz R, 2004: Cell specificity of aquaporins 0,3 , and 10 expressed in the testis, efferent ducts, and epididymis of adult rats. J Androl 25, 494-505.

Hermo L, Schellenberg M, Liu LY, Dayanandan B, Zhang T, Mandato CA, Smith CE, 2008: Membrane domain specificity in the spatial distribution of aquaporins 5 , 7,9 , and 11 in efferent ducts and epididymis of rats. J Histochem Cytochem 56, 1121-1135.

Huang HF, He RH, Sun CC, Zhang Y, Meng QX, Ma YY, 2006: Function of aquaporins in female and male reproductive systems. Hum Reprod Update 12, 785-795.

Lu DY, Li Y, Bi ZW, Yu HM, Li XJ, 2008: Expression and immunohistochemical localization of aquaporin-1 in male reproductive organs of the mouse. Anat Histol Embryol 37, 1-8.

Mota PC, Ramalho-Santos J, 2006: Comparison between different markers for sperm quality in the cat: Diff-Quik as a simple optical technique to assess changes in the DNA of feline epididymal sperm. Theriogenology 65, 1360-1375.

Munks MW, 2012: Progress in development of immunocontraceptive vaccines for permanent non-surgical sterilization of cats and dogs. Reprod Domest Anim 47(Suppl. 4), 223-227.

Nelson RD, Stricklett P, Gustafson C, Stevens A, Ausiello D, Brown D, Kohan DE, 1998: Expression of an AQP2 Cre recombinase transgene in kidney and male reproductive system of transgenic mice. Am J Physiol 275, C216-C226.

Nie R, Zhou Q, Jassim E, Saunders PT, Hess RA, 2002: Differential expression of estrogen receptors alpha and beta in the reproductive tracts of adult male dogs and cats. Biol Reprod 66, 1161-1168.

Oliveira CA, Carnes K, França LR, Hermo L, Hess RA, 2005: Aquaporin-1 and -9 are differentially regulated by oestrogen in the efferent ductule epithelium and initial segment of the epididymis. Biol Cell 97, 385-395.

Pastor-Soler N, Bagnis C, Sabolic I, Tyszkowski R, McKee M, Van Hoek A, Breton S, Brown D, 2001: Aquaporin 9 expression along the male reproductive tract. Biol Reprod 65, 384-393.

Pastor-Soler N, Isnard-Bagnis C, HerakKramberger C, Sabolic I, Van Hoek A, Brown D, Breton S, 2002: Expression of aquaporin 9 in the adult rat epididymal epithelium is modulated by androgens. Biol Reprod 66, 1716-1722.

Pastor-Soler NM, Fisher JS, Sharpe R, Hill E, Van Hoek A, Brown D, Breton S, 2010: Aquaporin 9 expression in the developing rat epididymis is modulated by steroid hormones. Reproduction 139, 613-621.

Picciarelli-Lima P, Oliveira AG, Reis AM, Kalapothakis E, Mahecha GA, Hess RA, Oliveira CA, 2006: Effects of 3-beta-diol, an androgen metabolite with intrinsic estrogen-like effects, in modulating the aquaporin-9 expression in the rat efferent ductules. Reprod Biol Endocrinol 4, 5161.

Schnermann J, Chou CL, Ma T, Traynor T, Knepper MA, Verkman AS, 1998: Defective proximal tubular fluid reabsorption in transgenic aquaporin-1 null mice. Proc Natl Acad Sci USA 95, 9660-9664.

Schön J, Neumann S, Wildt DE, Pukazhenthi BS, Jewgenow K, 2009: Localization of oestrogen receptors in the epididymis during sexual maturation of the domestic cat. Reprod Domest Anim 44(Suppl. 2), 294-301.

Scocco P, Aralla M, Catorci A, Belardinell C, Arrighi S, 2011: Immunodetection of aquaporin 5 in sheep salivary glands related to pasture vegetative cycle. Folia Histochem Cytobiol 49, 458-464.

Wrobel KH, Gurtler A, 2004: Morphology and innervation pattern of the feline urogenital junction. Anat Histol Embryol 33, 317-325.

Zhang D, Tan YJ, Qu F, Sheng JZ, Huang HF, 2012: Functions of water channels in male and female reproductive systems. Mol Aspects Med 33, 676-690.

Submitted: 26 Mar 2013; Accepted: 7 Jun 2013

Author's address (for correspondence) Silvana Arrighi, DVM, Laboratory of Anatomy, Department of Health, Animal Science and Food Safety, Università degli Studi di Milano, 2, Via Trentacoste, I-20134 Milano, Italy. E-mail: silvana.arrighi@unimi.it *Present address: Ospedale Veterinario San Michele, Via 1 Maggio 37, I-26838 Tavazzano con Villavesco (LO), Italy 